\title{
Improved $\mathrm{N}$ - and C-terminal sequencing of proteins by combining positive and negative ion MALDI in-source decay mass spectrometry
}

\author{
Simone Nicolardi ${ }^{a^{*}}$, David P.A. Kilgour ${ }^{b}$, Yuri E.M. van der Burgt ${ }^{a}$, Manfred Wuhrer ${ }^{a}$ \\ ${ }^{a}$ Center for Proteomics \& Metabolomics, Leiden University Medical Center, Leiden 2333 ZA, The \\ Netherlands.

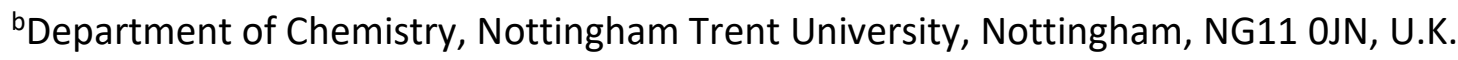

${ }^{*}$ Corresponding author: Simone Nicolardi

Leiden University Medical Center, Center for Proteomics and Metabolomics, Albinusdreef 2, 2333 ZA Leiden, The Netherlands. E-mail: s.nicolardi@lumc.nl 
TOC

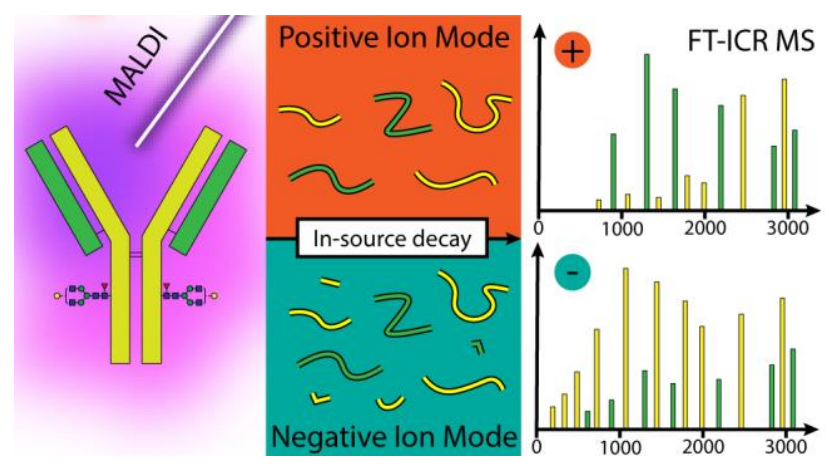




\begin{abstract}
(80-250 words)
The development of various ionization and fragmentation techniques has been of key importance for establishing mass spectrometry (MS) as a powerful tool for protein characterization. One example of this is matrix-assisted laser desorption/ionization (MALDI) combined with in-source decay (ISD) fragmentation that allows mapping of $\mathrm{N}$ - and C-terminal regions of large proteins without the need for proteolysis. Positive ion mode ISD fragments are commonly assigned in the mass region above $\mathrm{m} / \mathrm{z} 1000$, while MALDI matrix ions generally hamper the detection of smaller singly-charged fragments. The ultrahigh resolving power provided by Fourier transform ion cyclotron resonance (FT-ICR) MS partially overcomes this limitation, but to further increase detection of smaller fragments we have revisited the application of negative ion mode MALDI-ISD and found a good coverage of the peptide chain termini starting from $c^{\prime} 2$ and $z^{\prime} 2$ fragment ions. For the first time, we demonstrate that the combination of negative and positive ion MALDI FT-ICR MS is a useful tool to improve the characterization of mAbs. The different specificities of the two ion modes allowed us to selectively cover the sequence of the light and heavy chains of mAbs at increased sensitivity. A comprehensive evaluation of positive and negative ion mode MALDI-ISD FT-ICR MS in the $\mathrm{m} / \mathrm{z}$ range 46-13,500 showed an increased sequence coverage for three standard proteins, namely myoglobin, SiLuLite mAb, and NIST mAb. The data obtained in the two ion modes were, in part, complementary.
\end{abstract}




\section{Introduction}

Protein primary structure determination by mass spectrometry (MS) is performed with various combinations of ionization and fragmentation approaches. 1,2 Matrix-assisted laser desorption/ionization (MALDI) MS in combination with in-source decay (ISD) fragmentation may be used for relatively fast characterization of the $\mathrm{N}$ - and C-terminal regions of a protein sequence. ${ }^{3-7}$ Although MALDI-ISD MS is mainly known for the analysis of individual proteins, the characterization of mixtures of proteins or polypeptide chains is feasible without the need of upfront separation provided an instrument with ultrahigh resolving power is used. ${ }^{8,9}$ Recently, we have used the MALDI-ISD approach for the characterization of monoclonal antibodies and shown that MALDI-ISD Fourier transform ion cyclotron resonance (FT-ICR) provides complementary sequence information compared to other fragmentation-based MS techniques and allows for the localization of post-translational modification (PTMs) such as sulfation and glycation. ${ }^{10-12}$ One of the limitations of this approach, however, is that the detection of fragment ions with $\mathrm{m} / \mathrm{z}$-values lower than 1000 is hampered by the presence of the MALDI matrix clusters. ${ }^{13}$ Therefore, MALDI-ISD MS of proteins, either on time-of-flight (TOF) or FT ICR systems, is usually performed starting from $\mathrm{m} / \mathrm{z} 1000$ and consequently lacks sequence coverage of the terminal regions. Although this limitation can be tackled by the use of pseudo-MS ${ }^{3}$ analysis (or T3-sequencing), it is noted that this approach generally suffers from lower sensitivity due to the low isolation and fragmentation efficiencies in, for example, collision-induced dissociation (CID) experiments. ${ }^{14,15}$

MALDI-ISD protein fragmentation occurs both in positively and negatively charged species. ${ }^{16-19}$ The fragmentation mechanism of MALDI-ISD using reducing matrices involves the transfer of a hydrogen atom from the matrix molecule to the carbonyl group on the backbone of a protein. ${ }^{4,20,21}$ This event leads to the formation of radical ions which can fragment to generate $c^{\prime}$ and radical $z$ ions. These latter ion species can form $z, z^{\prime}$ and matrix-adducted $z$ ions. ${ }^{22,23}$ The intensity of MALDI-ISD fragment ions detected in either negative or positive ion mode is influenced by the presence of acidic or basic amino acid residues, respectively. ${ }^{6,24}$ The negative ionization mode is beneficial for the MALDI-ISD MS analysis of acidic peptides ${ }^{16,25-27}$ The larger 
fragment ions more likely include both acidic and basic residues and consequently show similar relative intensities in positive and negative ion mode MALDI-ISD MS although this latter, in general, provides a lower sensitivity. ${ }^{16,28,29}$ Since the amino acid sequence regions close to the protein termini may be biased towards basic or acidic residues, MALDI-ISD fragmentation in this region can result in different efficiencies of ionization, depending on the mode used. For example, Asakawa analyzed myoglobin by both positive and negative ion MALDI-ISD TOF MS and showed that fragment ions smaller than $\mathrm{m} / \mathrm{z} 1000$ were detected in negative ion mode to provide sequence information complementary to the positive ion mode analysis, resulting in an improved sequence coverage of $67 \% .{ }^{16}$

For the purpose of top-down MALDI-ISD MS analysis of mAbs simultaneous analysis of fragment ions from both the light and the heavy chains (LC and HC) is desirable. ${ }^{10}$ Since the termini of these mAb subunits generally exhibit different acidic/basic properties we hypothesized that positive and negative ion MALDI-ISD MS analyses may provide different results with regard to fragment ion intensities and sequence coverage. The aim of this study was to evaluate the complementarity of positive and negative ion MALDI-ISD FT-ICR MS, over a wide $\mathrm{m} / \mathrm{z}$ range, for the analysis of the primary protein structure, exemplified with horse myoglobin, SiLuLite mAb, and NIST MAb. 


\section{Material and Methods}

\section{Chemicals}

Myoglobin from equine skeletal muscle, 1,5-diaminonaphtalene (1,5-DAN; handle with care, check material safety data sheet), and acetonitrile (ACN; MS grade) were purchased from Sigma-Aldrich. Formic acid (FA) was purchased from Honeywell Fluka (USA). SiLuLite mAb (2 mg/ml ; IgG1lambda, CHO, Sigma) and NIST mAb (2 mg/mL; HzlgG1-kappa, NSO) standards were provided by the Consortium for Top-Down Proteomics (CTDP). ${ }^{10,30}$ A $2 \mathrm{mg} / \mathrm{mL}$ solution of myoglobin was prepared in water. SiLuLite $\mathrm{mAb}$ at $1 \mathrm{mg} / \mathrm{mL}$ in PBS, $\mathrm{pH}$ 6.8, was digested with 1000 units of the enzyme Fabricator (IdeS; Genovis, Lund, Sweden), according to the manufacturer's instructions, at $37{ }^{\circ} \mathrm{C}$ for $30 \mathrm{~min}$, under shaking (350 rpm). The reaction was quenched by adding trifluoroacetic acid (Honeywell Fluka, USA) to a final concentration of $\sim 0.05 \%$ and the samples were stored at $-80^{\circ} \mathrm{C}$ until further use.

SiLuLite mAb, SiLuLite mAb digest and NIST mAb required desalting using SPE-tips (ZipTip; Merck Millipore) filled with $0.6 \mu \mathrm{L}$ of $\mathrm{C} 18$ resin. Each tip was washed with a water/ACN (v/v 50\%:50\%) solution and equilibrated with water. Then, $2 \mu \mathrm{L}$ of mAb solution was allowed to bind to the SPE-tip by pipetting 20 times in and out. Finally, the loaded SPE-tip was flushed with water and the sample was eluted in $3 \mu \mathrm{L}$ of water/ACN (v/v 50\%:50\%).

Two saturated solutions of 1,5-DAN were prepared in water/ACN ( $/ / v$ 50\%:50\%) and in water/ACN/FA, 50\%:49.95\%:0.05\%, respectively.

\section{MALDI Spotting}

For the comparison of positive and negative ion mode spectra, the proteins standards ( $1 \mu \mathrm{L}$ of myoglobin or $3 \mu \mathrm{L}$ of C18-SPE mAb eluate) were spotted onto a ground steel MALDI target plate and mixed with $1 \mu \mathrm{L}$ of 1,5-DAN in water/ACN (v/v 50\%:50\%). For standard analysis in positive ion mode in the $\mathrm{m} / \mathrm{z}$-ranges $1012-13500$ and 1012-7000, SiLuLite mAb and SiLuLite mAb digest were diluted 1:10 with water and then spotted (1 $\mu \mathrm{L})$ using $1 \mu \mathrm{L}$ of 1,5-DAN in water/ACN/FA (v/v 50\%:49.95\%:0.05\%). The droplets were then allowed to dry at room temperature. 
MALDI-ISD FT-ICR Mass spectrometry

All MALDI-ISD MS experiments were performed on a $15 \mathrm{~T}$ solariX XR FT-ICR mass spectrometer (Bruker Daltonics) equipped with a CombiSource and a ParaCell. The MS system was operated using ftmsControl software (Bruker Daltonics). A Smartbeam-II laser system (Bruker Daltonics) was used at a frequency of $500 \mathrm{~Hz}$ and 200 laser shots per measurement. SiLuLite mAb was analyzed using a previously reported strategy based on positive ion mode acquisition in three different $m / z$-ranges: 1012-5000, 1012-7000, and 3495-30000. ${ }^{10}$ For the comparison of positive and negative ion mode spectra of the three proteins, the ion transmission was optimized in three additional $\mathrm{m} / \mathrm{z}$-ranges, namely 46-5000, 299-5000 and 506-5000. All transmission and MALDI parameters were kept constant when switching from positive to negative ion mode except for the polarity of the applied voltages. Fifteen scans were summed for each spectrum. The spectra in the $\mathrm{m} / \mathrm{z}$-range 46-5000 were acquired with $2 \mathrm{M}$ while the spectra in the $\mathrm{m} / \mathrm{z}$ ranges 299-5000 and 506-5000 were acquired with $1 \mathrm{M}$ data points. A positive ion mode spectrum of myoglobin was obtained in the $\mathrm{m} / \mathrm{z} 1012-7000$ from 12 scans while the corresponding negative ion mode spectrum was obtained from 30 scans. All data files were recorded to include the transient data (fid) file.

\section{Spectra visualization and processing}

Magnitude mode (mFT) spectra were visualized in DataAnalysis 5.0 SR1 (Bruker Daltonics). mFT and absorption mode (aFT) spectra were processed using AutoVectis (Spectroswiss, Lausanne, Switzerland) as previously reported. ${ }^{10,31-33}$ Additionally, theoretical $m / z$-values of ISD fragments were generated using the online ProteinProspector tool and the signal-to-noise ratio (S/N) of ISD fragment ions was measured using mMass software. ${ }^{34} 35$

Histograms plots were generated in Microsoft Excel 2016 and figures were made in Adobe Illustrator CC 2018. 


\section{Results and Discussion}

MALDI-ISD FT-ICR MS of horse myoglobin

Previously, we performed the structural characterization of horse myoglobin using positive ion MALDI-ISD FT-ICR MS in the $\mathrm{m} / \mathrm{z}$-range $1012-12000$. The resulting sequence coverage was $87 \%$.

8 In this study, we analyzed myoglobin in positive and negative ion mode. According to what previously reported by limuro and co-workers, ${ }^{29}$ positive and negative fragment ions showed similar profiles above $\mathrm{m} / \mathrm{z} 3000$ (Figure S1 and Figure S2) while large differences were observed in the lower $\mathrm{m} / \mathrm{z}$-range. Above $\mathrm{m} / \mathrm{z} 3000$, the negative ion mode analysis provides a lower sensitivity and more scans must be acquired to achieve the same signal intensity of the corresponding positive ion mode spectra (Figure S2). At $m / z$-values higher than 7000 , the negative ion mode gives low-quality spectra. The differences observed below $\mathrm{m} / \mathrm{z} 3000$ are in agreement with Asakawa and co-workers who showed that, due to differences in the amino acid composition of the $\mathrm{N}$ - and $\mathrm{C}$-terminal regions of myoglobin, the positive and the negative ion spectra, below $m / z 2000$, are different and that fragment ions smaller than $m / z 1000$ (i.e., up to $c^{\prime} 8$ and $z^{\prime} 8$ ) can be detected in negative mode. ${ }^{16}$

Myoglobin was further analyzed in the $m / z$-range 46-5000 and the intensity (i.e., signal-to-noise ratio, S/N) of $c^{\prime}$ and $z^{\prime}$ ions up to $m / z 3000$ were evaluated (Figure S3, Figure 1 and Figure 2). Fifteen summed spectra were acquired for each ionization mode. In the evaluated $\mathrm{m} / \mathrm{z}$-range, the first 27 residues (i.e., GLSDGEWQQVLNVWGKVEADIAGHGQE...) and the last 27 residues (i.e., ...AQGAMTKALELFRNDIAAKYKELGFQG) can be sequenced by the detection of singlycharged $c^{\prime}$ and $z^{\prime}$ ions, respectively (Table S1). In the mass spectra, the signal of the matrix clusters, of the heme $\left(\mathrm{C}_{34} \mathrm{H}_{32} \mathrm{FeN}_{4} \mathrm{O}_{4}\right)$ and its matrix adducts dominate over the $c^{\prime}$ and $z^{\prime}$ fragment ions (Figure 1 and Figure S3). However, the ultrahigh resolving powers provided by absorption mode visualization of the FT-ICR MS spectra allowed us to reliably distinguish the signal of the fragment ions from the matrix clusters and other ion species. In general, negative $c^{\prime}$ ions were detected at a higher signal intensity than positive ions while negative $z^{\prime}$ ions were more intense than positive $z^{\prime}$ ions only up to $z^{\prime} 14$ (Figure 1 and Figure 2). Starting from z'15, positive mode $z^{\prime}$ ions include Arg139 in their sequence. Of note, arginine residues are known to 
boost the intensity of positive ISD fragments, more than any other basic residue. ${ }^{36}$ This may explain the higher intensity of positive $z^{\prime} 15, z^{\prime} 16, z^{\prime} 17$, and $z^{\prime} 18$ compared to the corresponding negative fragment ions. Positive and negative $z^{\prime}$ ions from $z^{\prime} 19$ to $z^{\prime} 27$ were observed with similar $\mathrm{S} / \mathrm{N}$ values.

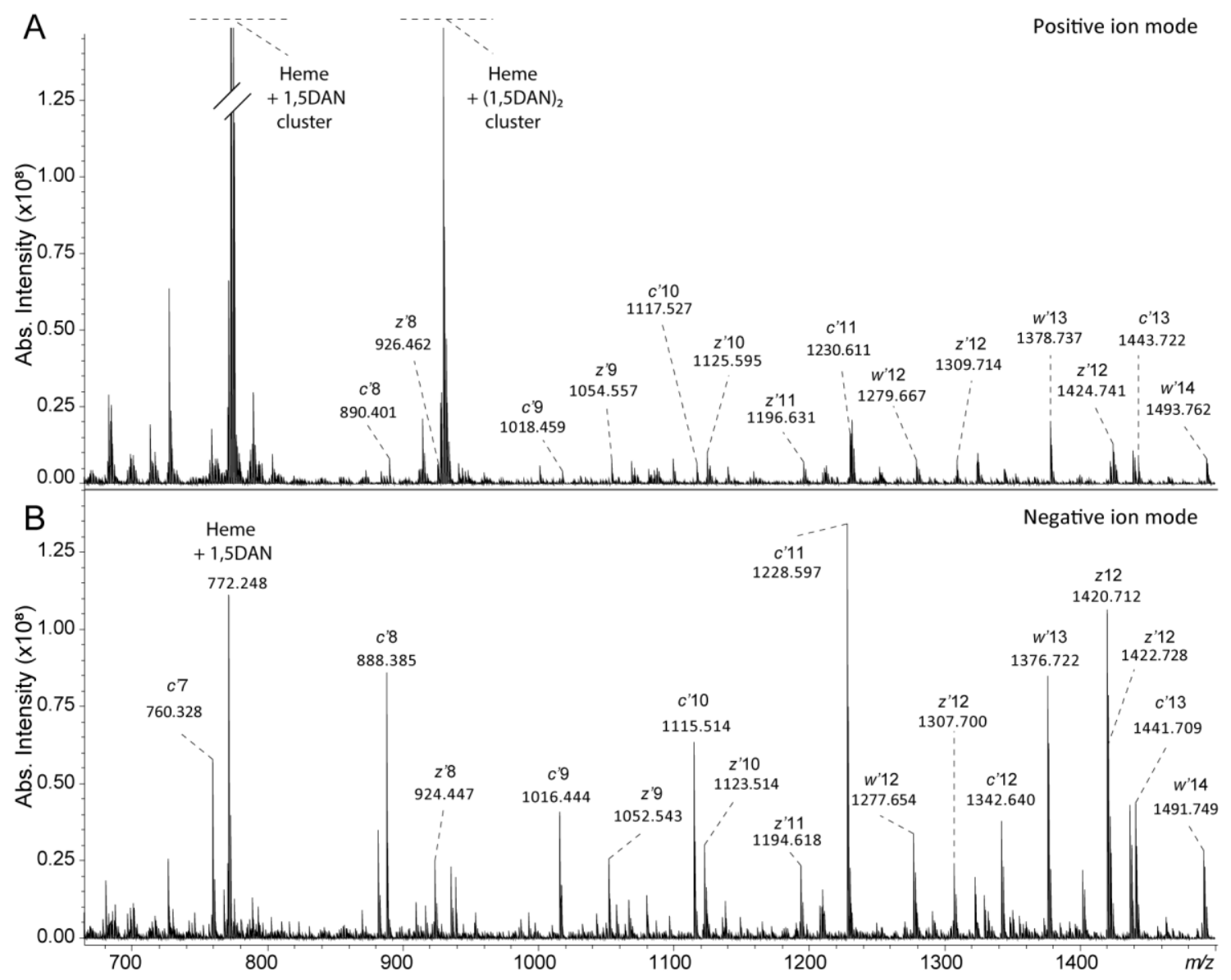

Figure 1. Enlargements of top-down A) positive and B) negative ion MALDI-ISD FT-ICR MS spectra of myoglobin. Negative $c^{\prime}$ and $z^{\prime}$ fragment ions were detected at a higher signal intensity than positive ions. Matrix clusters and matrix-adducted heme clusters were detected at a higher signal intensity in the positive ion mode (see also Figure S3). 

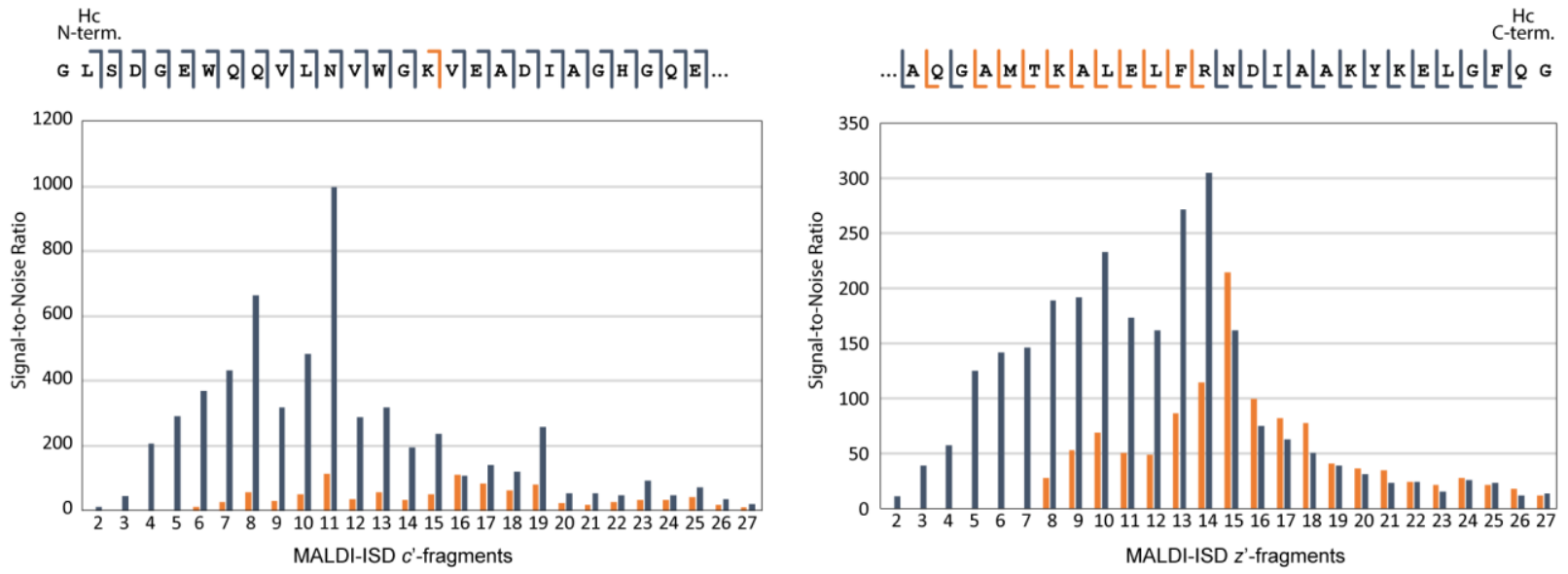

Figure 2: Overview of the distribution of $c^{\prime}$ and $z^{\prime}$ fragment ions of myoglobin analyzed by positive and negative ion MALDI-ISD FT-ICR MS (see Figure 1 ) in the $\mathrm{m} / \mathrm{z}$-range $46-3000$ (average of 5 replicates for each mode). Small fragment ions were more intense in negative ion mode than in positive mode with negative $c^{\prime}$ ions generally more intense than the corresponding positive ions over the entire $\mathrm{m} / \mathrm{z}$-range, while negative $z^{\prime}$ ions were more intense than positive ions only up to $z^{\prime} 14$. Note that the presence of Arg139 explains an inversion of the trend with $z^{\prime}$ ions up to $z^{\prime} 18$ more intense in positive ion mode. Larger positive and negative $z^{\prime}$ ions had similar intensity. Negative ion mode analysis allowed the detection of smaller $c^{\prime}$ (i.e., $c^{\prime} 2, c^{\prime} 3, c^{\prime} 4$, and $\left.c^{\prime} 5\right)$ and $z^{\prime}$ (i.e., $z^{\prime} 2, z^{\prime} 3, z^{\prime} 4, z^{\prime} 5, z^{\prime} 6$, and $\left.z^{\prime} 7\right)$. On average, the relative standard deviation of the $S / N$ values was $25 \%$ with a minimum of $7 \%$ and a maximum of $42 \%$.

Although fragment ions smaller than $\mathrm{m} / \mathrm{z} 1000$ were also detected in positive ion mode (starting from $c^{\prime} 6$ at $\mathrm{m} / z 576.262$ and $z^{\prime} 8$ at $\mathrm{m} / z$ 926.462), the intensity of negative fragment ions was clearly higher. The smallest negative $c^{\prime}$ and $z^{\prime}$ ions of myoglobin were detected at $\mathrm{m} / \mathrm{z}$ $186.1246\left(c^{\prime} 2\right)$ and $m / z 187.0751\left(z^{\prime} 2\right)$, respectively (Figure S4). This is an improvement compared to the smallest fragments of myoglobin (i.e., $c^{\prime} 8$ and $z^{\prime} 8$ ) previously detected by Asakawa and co-workers using negative ion MALDI-ISD FT-ICR MS. ${ }^{16}$ Ultrahigh field FT-ICR MS analysis allows resolving fragment ions with very low $\mathrm{m} / \mathrm{z}$-values, although at low sensitivity due to inherent technological limitations. It is worth noting that alternative fragmentation techniques often suffer from a low sensitivity from small fragment ions thus, a combination of 
positive and negative ion MALDI-ISD FT-ICR MS can provide more information on the termini compared to other techniques. ${ }^{37,38}$

Compared to our previous study, ${ }^{8}$ the combination of positive and negative MALDI-ISD FT-ICR MS measurements resulted in extended sequence coverage towards the $\mathrm{N}$ - and $\mathrm{C}$-termini and increased the total sequence coverage of myoglobin from $87 \%$ to $99 \%$ (Figure 3).

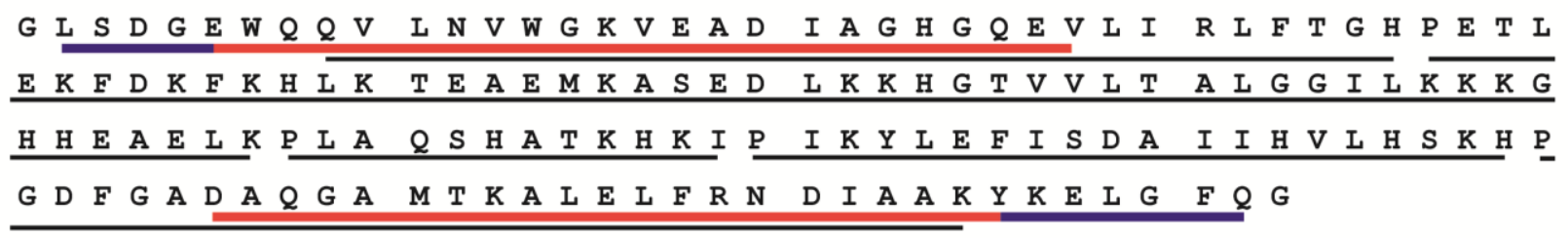

Figure 3. Sequence coverage of myoglobin analyzed by top-down MALDI-ISD FT-ICR MS. The black line indicates the sequence coverage (87\%) obtained from the positive ion mode analysis in the $m / z$-range $1012-12000$ as reported previously. ${ }^{8}$ The sequence information obtained by positive ion mode analysis in the $\mathrm{m} / \mathrm{z}$-range $46-3000$ is indicated by the red line while the additional sequence coverage obtained by negative ion mode analysis in the same $\mathrm{m} / \mathrm{z}$-range is highlighted by the blue line. Negative ion mode analysis resulted in the detection of smaller fragment ions that allowed extending the sequence coverage further towards the termini. The combination of positive and negative ion mode analysis yielded a total sequence coverage of $99 \%$.

MALDI-ISD FT-ICR MS of SiLuLite mAb

Recently, we reported a strategy for the characterization of mAbs based on positive ion MALDIISD FT-ICR MS. ${ }^{10}$ This strategy, that involves the measurements of ISD fragment ions in the $\mathrm{m} / \mathrm{z}$ ranges 1012-13500 and 1012-7000, was used for the characterization of intact and IdeS digested SiLuLite mAb (Figures S5 and S6). Sequence coverage of 38\% and 51\% were obtained for Hc and Lc, respectively (Figure 4). Due to the selected $m / z$ range, the first 9-12 residues of the termini of $\mathrm{Hc}, \mathrm{Lc}, \mathrm{Fc} / 2$, and $\mathrm{Fd}^{\prime}$ were not sequenced. To further increase the sequence 
coverage these mAb subunits were also analyzed by positive and negative MALDI-ISD FT-ICR MS in the $m / z$-range $46-5000$ and $c^{\prime}$ and $z^{\prime}$ fragment ions were evaluated up to $m / z 3000$. This additional analysis resulted in a $\mathrm{Hc}$ and Lc sequence coverage of $45 \%$ and $55 \%$, respectively (Figure 4).

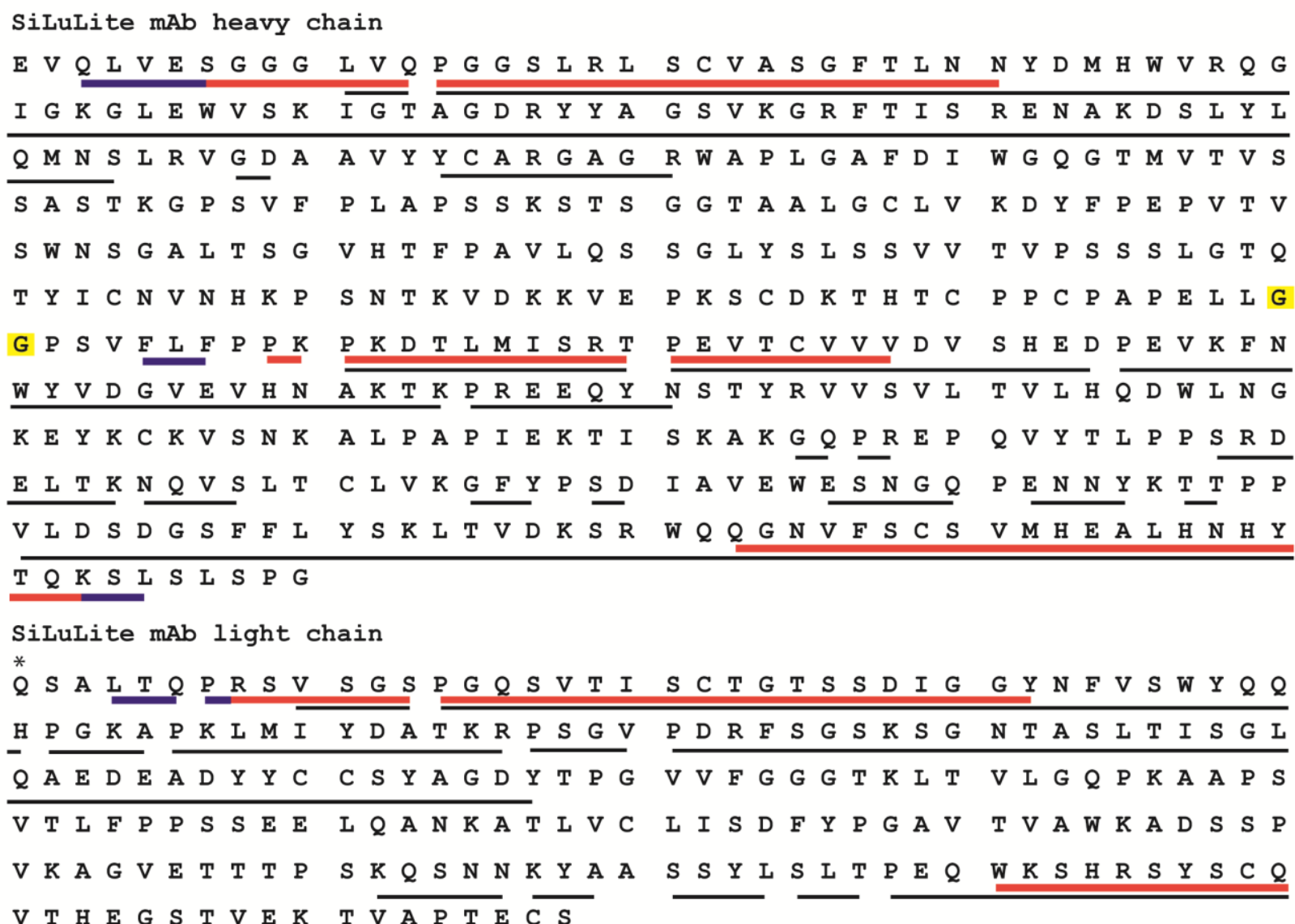

Figure 4. Sequence coverage of SiLuLite mAb analyzed by top-down and middle-down MALDIISD FT-ICR MS analysis. The black line indicates the sequence coverage obtained from the analysis in the $\mathrm{m} / \mathrm{z}$-range $1012-13500$ for the intact $\mathrm{mAb}$ and the $\mathrm{m} / \mathrm{z}$-range $1012-7000$ for the IdeS-digested mAb. This was 38\% and $51 \%$ for $\mathrm{Hc}$ and Lc, respectively. The sequence coverage obtained by positive ion mode analysis in the $\mathrm{m} / \mathrm{z}$-range $46-3000$ is indicated in red while the additional sequence information obtained by negative ion mode analysis, in the same $\mathrm{m} / \mathrm{z}$ range, is highlighted in blue. The combination of positive and negative ion MALDI-ISD FT-ICR MS 
analysis in the $\mathrm{m} / \mathrm{z}$-range lower than 1000 , allowed extending the sequence coverage to $45 \%$ and $55 \%$ for $\mathrm{Hc}$ and $\mathrm{LC}$, respectively. * indicates a pyroGlu residue.

Fragment ions detected in $\mathrm{m} / \mathrm{z}$-range 46-3000 were generated from sequence portions with different acid/basic characteristics (Tables S2 and S3), consequently, for the analysis of intact SiLuLite mAb, clear differences were observed between the positive and the negative ion mode spectra with the $c^{\prime}$ ions of $\mathrm{Hc}$ being the most intense species in the positive ion spectra and the $c^{\prime}$ ions of the Lc being the most intense species in the negative ion spectra (Figure 5). The evaluation of the intensities of positive and negative $c^{\prime}$ and $z^{\prime}$ ions is summarized in Figure 6.

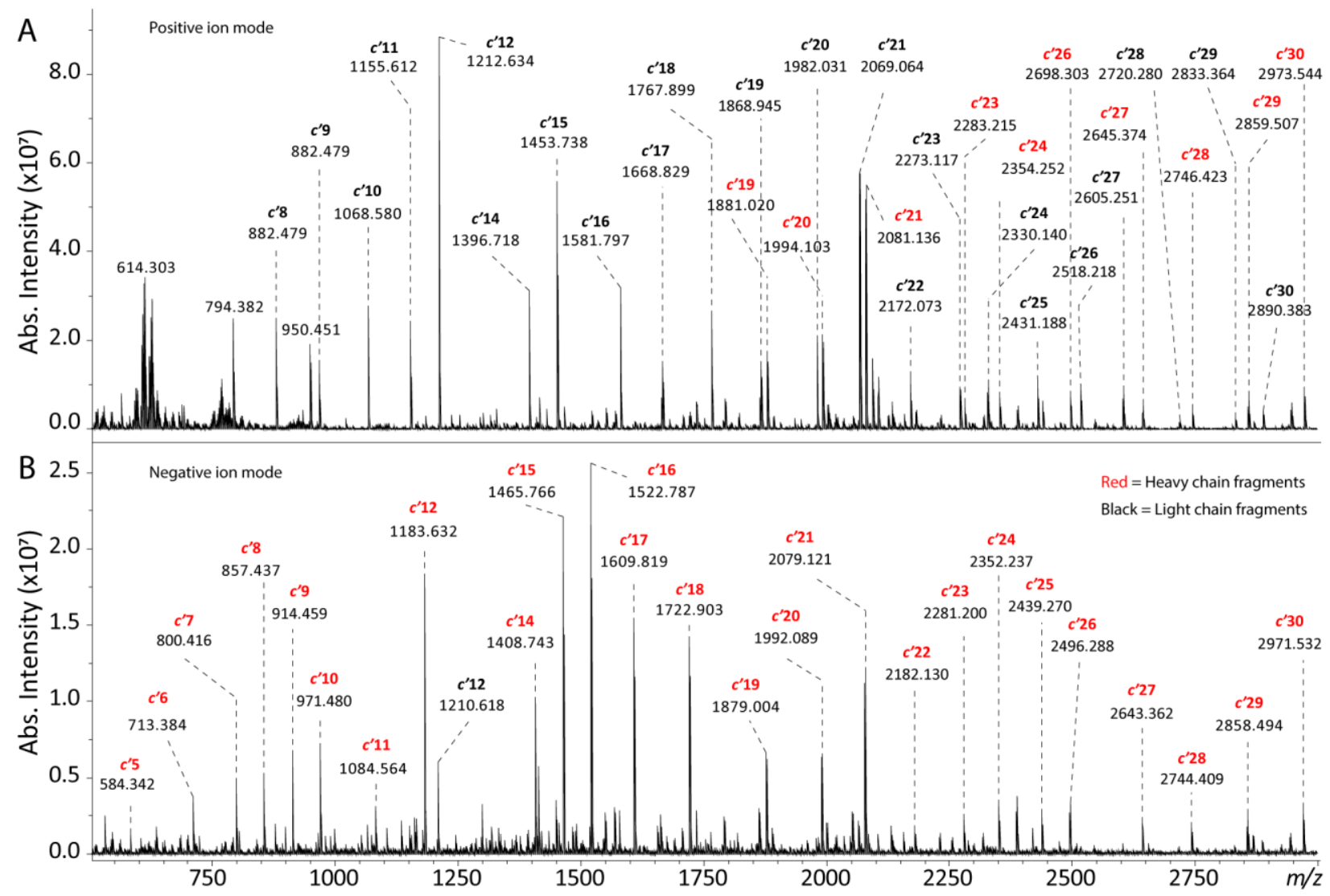

Figure 5. Enlargements of top-down A) positive and B) negative ion MALDI-ISD FT-ICR MS spectra of intact SiLuLite mAb. The different acidic/basic characteristics of the terminal regions of $\mathrm{LC}$ and $\mathrm{Hc}$ led to different distributions of fragment ions in the two ionization modes with $c^{\prime}$ 
fragment ions of Lc being the most intense in positive ion mode and $c^{\prime}$ fragment ions of $\mathrm{Hc}$ being the most intense in negative ion mode.
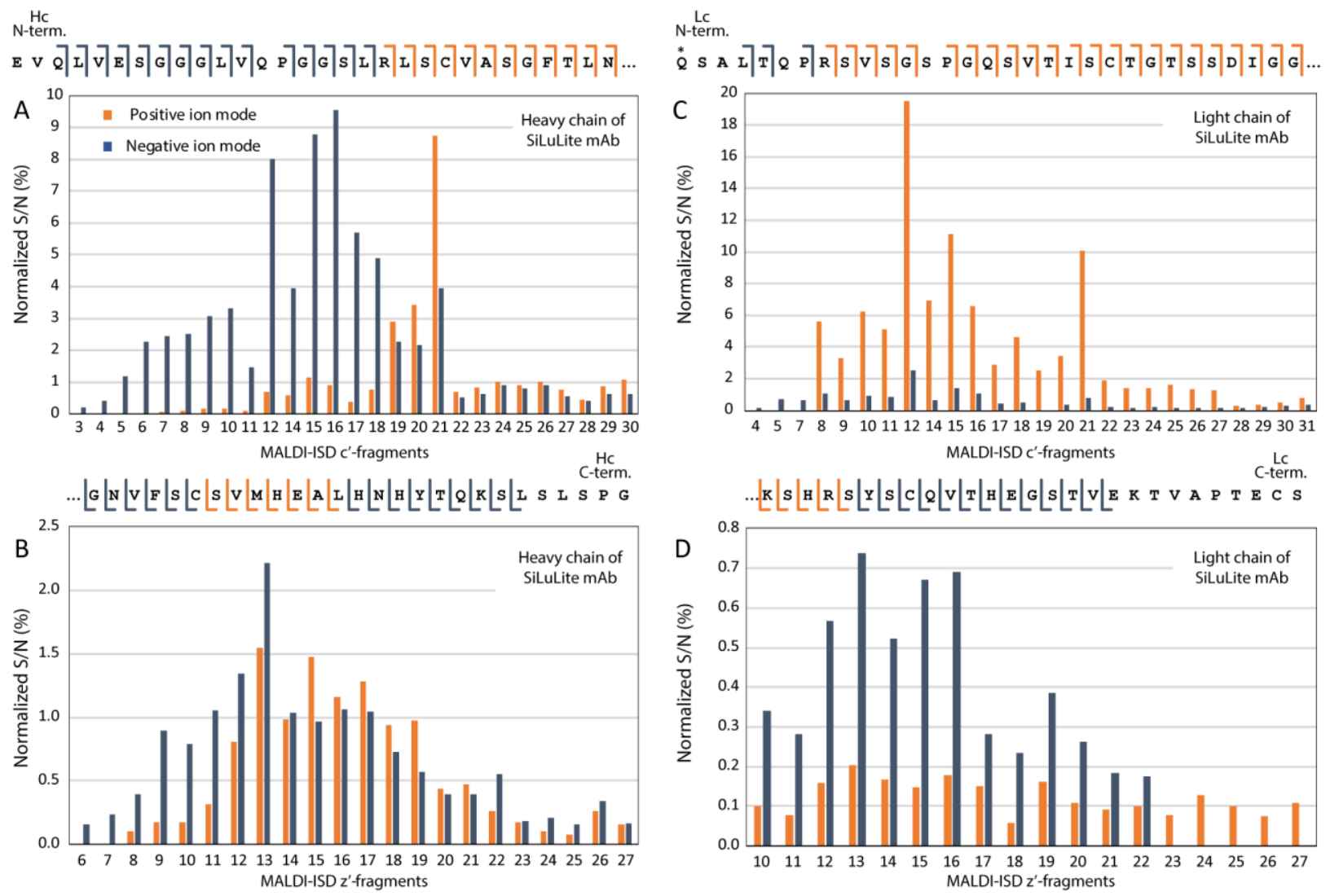

Figure 6. Overview of the distribution of fragment ions of LC and Hc of SiLuLite mAb generated by top-down positive and negative ion MALDI-ISD FT-ICR MS. Fragment ions from acidic regions were detected at a higher signal intensity (i.e. $S / N$ ) in negative ion mode (A and D). Fragment ions that include an arginine residue, rather than other basic residues, were detected at a higher signal intensity in positive ion mode ( $A, C$ and $D)$. In general, negative ion detection allowed the identification of smaller fragment ions even below $m / z 1000$ (A, B, C, and D). * indicates a pyroQ residue.

For all sequences, it was found that negative ion mode analysis allowed the identification of small fragments that were missed in positive ion mode. The presence of acidic amino acid residues in the $\mathrm{N}$-terminal region of the $\mathrm{Hc}$ (i.e. Glu1 and Glu6) contributed to the generation of 
negative $c^{\prime}$ ions with signal intensities higher than the corresponding positive ions. Interestingly, the positive $c^{\prime}$ ions of $\mathrm{Hc}$ became more intense than the negative ions only when Arg19 was present in the fragment sequence (i.e. for $c^{\prime} \geq 19$ ). The $\mathrm{N}$-terminal region of the $\mathrm{Lc}$, instead, lacks negatively charged amino acids up to Asp 28. Here, negative $c^{\prime}$ ions were more intense than positive ions only up to $c^{\prime} 7$. Then, the presence of Arg8 led to the generation of intense positive ions which dominate the spectra. The $z^{\prime}$ ions of both $\mathrm{Hc}$ and Lc were detected at a lower intensity compared to the $c^{\prime}$ ions. The C-terminal region of the Hc includes more basic residues (His433, His437, His439, and Lys443) than acidic residues (i.e. C-terminus, and Glu434) however, negative ions were more intense than corresponding positive ions not only for small fragments but also for some large fragments. The C-terminal region of the Lc contains four acidic residues (i.e. C-terminus, Glu204, Glu209, and Glu216) and five basic residues (Lys192, His194, Arg195, His203, and Lys210). Although the presence of the C-terminus and Glu434 could potentially lead to the generation of very small fragment ions (e.g. $z^{\prime} 3$ ), the series of negative $z^{\prime}$ ions of Lc only started from $z^{\prime} 10$ indicating that other factors, rather the only presence or absence of acidic amino acid residues, play a role in the generation of negative fragment ions. This has been previously shown for the negative ion mode analysis of acidic peptides where the influence of the acidity or basicity of amino acid residues was less straightforward than in positive ion mode. ${ }^{24,36}$

Different distributions of $c^{\prime}$ ions of $\mathrm{Fc} / 2$ were obtained from the analysis of Ides-digested SiLuLite mAb in positive and negative ion modes (Figure S7 and Figure S8). The $\mathrm{N}$-terminal region of Fc/2 does not contain acidic residues, however, negative $c^{\prime} 5, c^{\prime} 6$, and $c^{\prime} 9$ fragment ions were detected, while the equivalent positive ions were not. The increased sensitivity for small fragment ions in negative ion mode was attributed to the suppressed signal of the MALDI matrix clusters. It is noted that $z^{\prime}$ fragment ions of Fd' subunit were detected neither in positive nor in negative mode. 
In our previous report, intact NIST mAb was analyzed in positive ion mode by top-down MALDIISD FT-ICR MS in the $m / z$-range 1012-13500. ${ }^{10}$ The resulting sequence coverage was $31 \%$ and $65 \%$ for $\mathrm{Hc}$ and Lc, respectively (Figure S9). The analysis performed in this study, in the $\mathrm{m} / \mathrm{z}$ range 46-5,000 (see Table S4, Figure S10 and Figure S11), resulted in an increased Hc and LC sequence coverage of $33 \%$ and $70 \%$, respectively (Figure S9).

Interestingly, in contrast to what was observed for SiLuLite mAb, the $c^{\prime}$ ions of the Hc were more intense in the positive ion mode spectrum while $c^{\prime}$ ions of the Lc were more intense in the negative ion mode spectrum. For this $m A b$, the advantage of using the negative ion ISD over the positive ISD was limited to the $\mathrm{N}$-terminal region of the $\mathrm{LC}$ and the C-terminal region of $\mathrm{Hc}$. Notably, the evaluation of smaller fragments in positive ion ISD resulted in the improved characterization of the $\mathrm{N}$-terminal region of $\mathrm{Hc}$ and the $\mathrm{C}$-terminal region of the $\mathrm{Lc}$. The $\mathrm{N}$ terminal region of the $\mathrm{Hc}$ includes one acidic residue (Glu6) and two basic residues (Arg5 and Lys13), with pyroGlu as the $\mathrm{N}$-terminal residue. For $\mathrm{Hc}$, the positive $c^{\prime}$ fragments were more intense than the corresponding negative species probably due to the presence of an arginine residue at position five. On the contrary, the $\mathrm{N}$-terminal region of the $\mathrm{LC}$ includes two acidic residues (Asp1 and Asp27) and three acidic residues ( $N$-terminus, Arg18, and Arg28). Hence, for Lc the negative $c^{\prime}$ fragment ions were more intense up to $c^{\prime} 17$, after which the presence of Arg18 contributed to the generation of positive $c^{\prime}$ ions with higher intensities.

Similar to myoglobin and SiLuLite mAb, the $z^{\prime}$ fragment ions of NIST mAb were detected at a lower signal intensity than the $c^{\prime}$ ions. The C-terminal regions of the Hc of SiLuLite mAb and NIST mAb are identical. However, for NIST mAb, all negative $z^{\prime}$ ions of Hc were more intense than the corresponding positive $z^{\prime}$ ions. The C-terminal region of LC of NIST mAb is less acidic than the Lc of SiLuLite mAb and it includes an arginine residue at position 210 which could potentially lead to the observation of $z^{\prime} 3$ ion. The presence of Arg210 apparently influenced the intensity of positive $z^{\prime}$ ions which were observed at a higher intensity than the negative ions, however, the $z^{\prime} 3$ ion was not detected and the fragment series started from a higher position (i.e. $z^{\prime} 8$ ). 
Compared to the positive ion mode analysis, the negative ion MALDI-ISD FT-ICR MS analysis of NIST mAb provided additional structural information for the C-terminal region of $\mathrm{Hc}$ and the $\mathrm{N}$ terminal region of Lc.

The intensity of the matrix cluster ions was higher in the positive spectra, similar to the earlier observations during the analysis of SiLuLite mAb. However, small positive $c^{\prime}$ ions of Hc were detected in the matrix region indicating that the acidic/basic characteristic of the termini (e.g. the presence of arginine residues) of the protein may play a more important role in the generation of small fragment ions. 


\section{Conclusions}

In this study, we revisited MALDI-ISD MS as a fast characterization approach for sequence analysis of proteins. We combined positive and negative ion MALDI-ISD FT-ICR MS analyses to extend the sequence coverage of proteins towards their termini. The study was focused on the analysis of relatively small fragment ions, typically below $m / z 3,000$, and as a proof-of-principle, ISD fragment ions of horse myoglobin, SiLuLite mAb, and NIST mAb were evaluated in the $\mathrm{m} / \mathrm{z}$ range $46-3,000$. It should be noted that this study is the first report on the characterization of intact mAbs by negative mode MALDI-ISD FT-ICR MS and its combination with positive mode analysis. It was found that ISD fragment ions generated from acidic terminal regions of proteins were detected at a higher signal intensity in negative ion mode and even below $\mathrm{m} / \mathrm{z} 1,000$. Fragment ions that included an arginine residue rather than other basic residues were consistently detected at an increased signal intensity in positive ion mode. The specificity of positive and negative ion MALDI-ISD FT-ICR MS for basic and acidic terminal regions of proteins was particularly beneficial for the analysis of intact mAbs. The application of the two ionization modes provides increased sensitivity for ISD fragment ions of terminal regions of LC or HC according to their acidic/basic properties.

For all three proteins, it was found that negative ion mode spectra yielded sequence information that complements positive ion mode data. In addition, the detection of negative fragment ions at higher signal intensity ensured higher confidence for the identifications in the $m / z$-region densely populated by MALDI matrix ions. Differences were also observed for the matrix clusters which were more intense in positive mode. The lower intensity of the matrix cluster in the negative ion spectra may contribute to the higher sensitivity for fragment ions in the low $\mathrm{m} / \mathrm{z}$-region. It is noted that this phenomenon is not comprehensively understood and different factors, such as the role of the matrix molecules in the secondary reactions in the ionization mechanism, may be involved. Since the acidic/basic properties of the MALDI matrix have an effect on both the ionization and generation of fragment ions, further research is warranted to understand the influence of these properties on the formation of positive and negative fragment ions. 
In conclusion, we demonstrated that the combination of positive and negative ion MALDI-ISD FT-ICR MS improved the N- and C-terminal sequencing of proteins by tuning the sensitivity for fragment ions with specific acidic/basic characteristics and by allowing the detection of fragment ions in the $m / z$-region of the MALDI matrix clusters (i.e., $m / z \leq 1000$ ).

A comprehensive analysis of mAbs requires a multi-method approach for structural characterization. We use the relatively simple positive and negative ion MALDI-ISD strategy described in this manuscript, as part of these multi-method approaches, to provide additional sequence information on this important class of pharmaceutical proteins.

\section{Supporting Information}

Figure S1: MALDI-ISD FT-ICR MS spectra of myoglobin ( $m / z$-range 1012-7000). Figure S2: MALDI-ISD FTICR MS spectra of myoglobin ( $\mathrm{m} / \mathrm{z}$-range 3000-5000). Figure S3: MALDI-ISD FT-ICR MS spectra of myoglobin ( $m / z$-ranges 45-5000). Figure S4: Observed $c^{\prime} 2, c^{\prime} 3, z^{\prime} 2$ and $z^{\prime} 3$ fragment ions of myoglobin. Figure S5: MALDI-ISD MS spectrum of intact SiLuLite mAb. Figure S6: MALDI-ISD MS spectrum of IdeSdigested SiLuLite mAb. Figure S7: MALDI-ISD FT-ICR MS spectra of IdeS-digested SiLuLite mAb $\mathrm{m} / \mathrm{z} 500$ 3000. Figure S8: Distribution of $c^{\prime}$ fragment ions of Fc/2 of SiLuLite mAb. Figure S9: Sequence coverage of NIST mAb. Figure S10: Enlargements of MALDI-ISD FT-ICR MS spectra of intact NIST mAb. Figure S11: Overview of the distribution of fragment ions of LC and Hc of NIST mAb. Table S1: Theoretical MALDI-ISD fragment ions of myoglobin. Table S2: Theoretical MALDI-ISD fragment ions of SiLuLite mAb. Table S3: Theoretical MALDI-ISD fragment ions of Fc/2 SiLuLite mAb. Table S4: Theoretical MALDI-ISD fragment ions of NIST mAb. 


\section{References}

(1) Brodbelt, J. S. Anal. Chem. 2016, 88, 30-51.

(2) Macias, L. A.; Santos, I. C.; Brodbelt, J. S. Anal. Chem. 2019.

(3) Brown, R. S.; Lennon, J. J. Anal. Chem. 1995, 67, 3990-3999.

(4) Asakawa, D. Mass Spectrom. Rev. 2016, 35, 535-556.

(5) Hempel, B.-F.; Damm, M.; Mrinalini; Göçmen, B.; Karış, M.; Nalbantsoy, A.; Kini, R. M.; Süssmuth, R.

D. J. Proteome Res. 2020, 19, 1731-1749.

(6) Takayama, M.; Tsugita, A. Electrophoresis 2000, 21, 1670-1677.

(7) Hardouin, J. Mass Spectrom. Rev. 2007, 26, 672-682.

(8) Nicolardi, S.; Switzar, L.; Deelder, A. M.; Palmblad, M.; van der Burgt, Y. E. M. Anal. Chem. 2015, 87, 3429-3437.

(9) Tran, B. Q.; Barton, C.; Feng, J.; Sandjong, A.; Yoon, S. H.; Awasthi, S.; Liang, T.; Khan, M. M.; Kilgour, D. P. A.; Goodlett, D. R.; Goo, Y. A. Data Brief 2015, 6, 68-76.

(10) van der Burgt, Y. E. M.; Kilgour, D. P. A.; Tsybin, Y. O.; Srzentic, K.; Fornelli, L.; Beck, A.; Wuhrer, M.; Nicolardi, S. Anal. Chem. 2019, 91, 2079-2085.

(11) Tyshchuk, O.; Gstottner, C.; Funk, D.; Nicolardi, S.; Frost, S.; Klostermann, S.; Becker, T.; Jolkver, E.; Schumacher, F.; Koller, C. F.; Volger, H. R.; Wuhrer, M.; Bulau, P.; Molhoj, M. mAbs 2019, 11, 1219-1232.

(12) Gstottner, C.; Reusch, D.; Haberger, M.; Dragan, I.; Van Veelen, P.; Kilgour, D. P. A.; Tsybin, Y. O.; van der Burgt, Y. E. M.; Wuhrer, M.; Nicolardi, S. mAbs 2020, 12, 1682403.

(13) Asakawa, D.; Calligaris, D.; Zimmerman, T. A.; Pauw, E. D. Anal. Chem. 2013, 85, 7809-7817.

(14) Suckau, D.; Resemann, A. Anal. Chem. 2003, 75, 5817-5824.

(15) Asakawa, D.; Smargiasso, N.; De Pauw, E. Anal. Chem. 2014, 86, 2451-2457.

(16) Asakawa, D.; Smargiasso, N.; De Pauw, E. J. Am. Soc. Mass Spectrom. 2013, 24, 297-300.

(17) Takayama, M. ACS Omega 2019, 4, 20308-20314.

(18) Demeure, K.; Quinton, L.; Gabelica, V.; De Pauw, E. Anal. Chem. 2007, 79, 8678-8685.

(19) Soltwisch, J.; Dreisewerd, K. Anal. Chem. 2010, 82, 5628-5635.

(20) Kocher, T.; Engstrom, A.; Zubarev, R. A. Anal. Chem. 2005, 77, 172-177.

(21) Takayama, M. J. Am. Soc. Mass Spectrom. 2001, 12, 1044-1049.

(22) Nicolardi, S.; Kilgour, D. P. A.; Dolezal, N.; Drijfhout, J. W.; Wuhrer, M.; van der Burgt, Y. E. M. Anal. Chem. 2020, 92, 5871-5881.

(23) Asakawa, D.; Takahashi, H.; Iwamoto, S.; Tanaka, K. Phys. Chem. Chem. Phys. 2018, 20, 1305713067.

(24) Takayama, M.; Tsugita, A. Int. J. Mass Spectrom. 1998, 181, L1-L6.

(25) McMillen, C. L.; Wright, P. M.; Cassady, C. J. J. Am. Soc. Mass Spectrom. 2016, 27, 847-855.

(26) Asakawa, D. Mass Spectrom (Tokyo) 2013, 2, A0019.

(27) Smargiasso, N.; Quinton, L.; De Pauw, E. J. Am. Soc. Mass Spectrom. 2012, 23, 469-474.

(28) Takayama, M.; Sekiya, S.; limuro, R.; Iwamoto, S.; Tanaka, K. J. Am. Soc. Mass Spectrom. 2014, 25, 120-131.

(29) limuro, R.; Takayama, M. Mass Spectrom. (Tokyo) 2014, 3, S0023-S0023.

(30) Srzentic, K.; Fornelli, L.; Tsybin, Y.; Loo, J.; Agar, J.; Chamot-Rooke, J.; Danis, P.; Ge, Y.; Goodlett, D.; Kelleher, N.; Pasa Tolic, L.; Smith, L.; Toby, T.; Nagornov, K.; Brodbelt, J.; Greer, S.; Dupré, M.; Clarke, D.; Lin, Z.; Haselmann, K., et al. 66th ASMS Conference on Mass Spectrometry and Allied Topics 2018 San Diego 2018, Poster ID 294106.

(31) Kilgour, D. P. A.; Wills, R.; Qi, Y.; O'Connor, P. B. Anal. Chem. 2013, 85, 3903-3911.

(32) Kilgour, D. P.; Neal, M. J.; Soulby, A. J.; O'Connor, P. B. Rapid Commun. Mass Spectrom. 2013, 27, 1977-1982. 
(33) Kilgour, D. P.; Van Orden, S. L. Rapid Commun. Mass Spectrom. 2015, 29, 1009-1018.

(34) http://prospector.ucsf.edu/prospector/mshome.htm.

(35) Strohalm, M.; Hassman, M.; Košata, B.; Kodíček, M. Rapid Commun. Mass Spectrom. 2008, 22, 905908.

(36) Demeure, K.; Gabelica, V.; De Pauw, E. A. J. Am. Soc. Mass Spectrom. 2010, 21, 1906-1917.

(37) Vincent, D.; Binos, S.; Rochfort, S.; Spangenberg, G. Proteomes 2019, 7, 33.

(38) Fort, K. L.; Cramer, C. N.; Voinov, V. G.; Vasil'ev, Y. V.; Lopez, N. I.; Beckman, J. S.; Heck, A. J. R. J. Proteome Res. 2018, 17, 926-933. 